\title{
First-level data processing for the CORONAS-F/SPIRIT EUV experiment
}

\author{
S. Bogachev ${ }^{1}$, V. Slemzin ${ }^{2}$, O. Bugaenko ${ }^{1}$, V. Grechnev ${ }^{3}$, \\ S. Bozhenkov ${ }^{2}$, S. Kuzin ${ }^{2}$, I. Zhitnik ${ }^{2}$ and A. Pertsov ${ }^{2}$ \\ ${ }^{1}$ Sternberg Astronomical Institute, Moscow State University \\ ${ }^{2}$ Lebedev Physical Institute of RAS \\ ${ }^{3}$ Institute of Solar-Terrestrial Physics, RAS
}

\begin{abstract}
More than 300000 images of the Sun have been acquired during the SPIRIT experiment with 10 telescopic and spectroheliographic EUV and X-ray instruments aboard the CORONAS-F satellite. We have developed methods and routines for the first-level data processing to make the SPIRIT data easy for use in a way similar to that one for SOHO/EIT images.
\end{abstract}

The CORONAS-F is an international project of the cooperation between Russian and Ukrainian Space Agencies and Academies of Sciences with a participation of other countries. It operates in the Earth's orbit since August 2001. The SPectroheliographIc $\mathrm{X}$-Ray Imaging Telescope (SPIRIT) aboard the CORONAS-F spacecraft provides imaging of the Sun in 6 telescopic and 4 spectroheliographic channels.

SPIRIT data archives contain more than 300000 images and spectra of total $>100$ Gbytes. SPIRIT data currently exist in the following formats:

- raw data in the initial telemetry format,

- level 0 SPIRIT/FITS format containing non-processed images and spectra extracted from telemetry files,

- level 1 processed FITS files standardized with the EIT data format,

- pictures (JPEG, PNG); AVI and MPEG movies, and

- catalogs of images, spectra, and auxiliary flight data.

We endeavor to standardize the EUV SPIRIT data to make their analysis easy for any researcher. To solve this task, we transform level 0 to level 1 data files, which have as much similarity as possible with data files produced by instruments well known for the solar community, e.g., SOHO/EIT files.

Features of the SPIRIT data processing are determined by properties of the SPIRIT instruments. For example, particularities of the CORONAS-F attitude system require the centering images and compensation for the roll angle. Therefore, in the preparation of the properly aligned images, we have to use some special methods in addition to the information obtained from the starguide cameras.

Certainly, the best way for any data processing would be the minimal intervention into data to avoid any losses of information. However, large instrumental contributions, varying roll angle and image centering make their usage difficult. Therefore, we admit some data losses, but the data become easy to use and can be handled farther in a way similar to that one for SOHO/EIT files. This seems reasonable, because EUV channels of the SPIRIT and EIT are very similar. This way facilitates joint analysis of images acquired with both telescopes. 


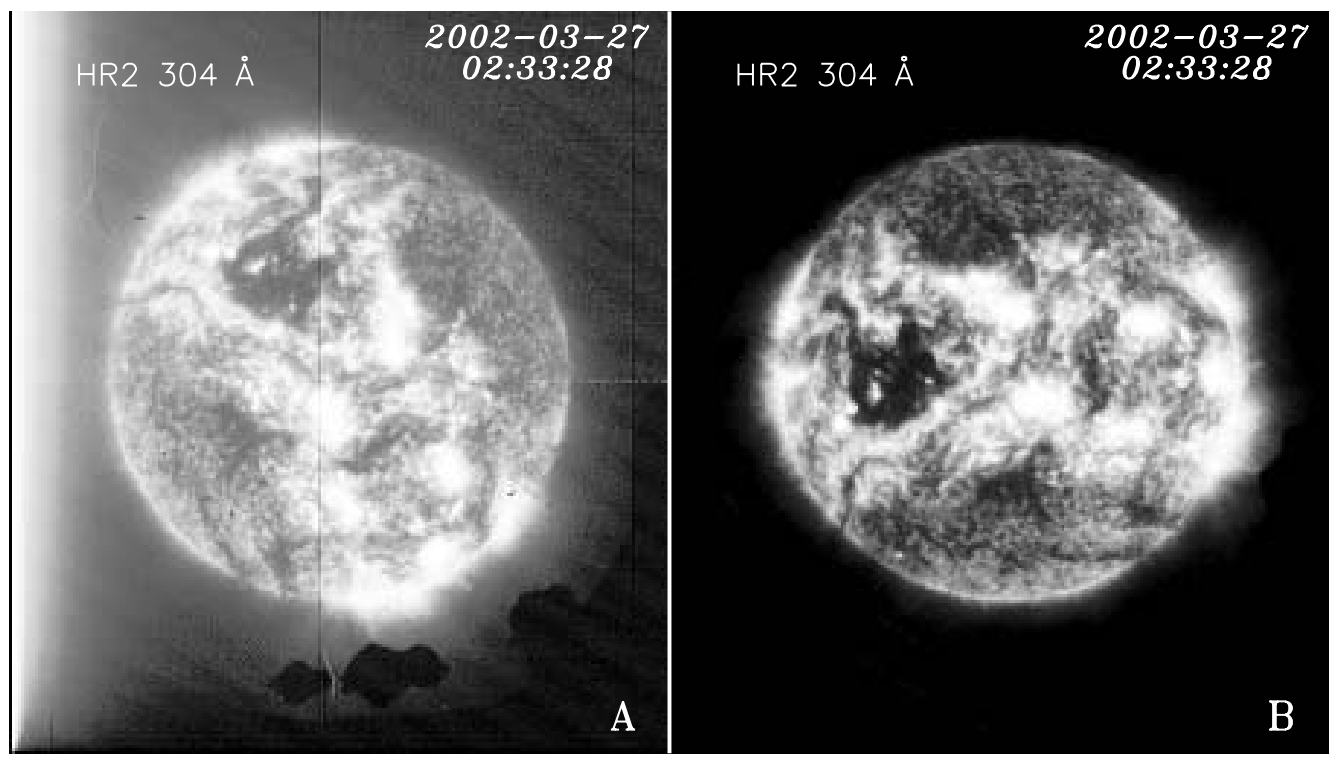

Figure 1. An example of the initial image (A) and the Level 1 processed image (B).

Some inevitable data losses occur in this way, but raw telemetry data files are strongly preserved. Hence, if some special data handling is required, one can retrieve the data from raw files and process them in a proper way.

Currently, Level 1 procedures provide for (we demonstrate initial and processed images in Figure 1):

- background subtraction and flat-field correction,

- correction of the detector nonlinearity,

- correction of the roll angle and centering,

- accurate coalignment of data cubes,

- restoration of lost pixels, and

- suppression of the 'snow' produced by energetic particles in radiation belts and polar caps.

Further analysis will allow improving the accuracy and quality of data processing. Nevertheless, it is important to introduce the SPIRIT data into handling and analysis as soon as possible. The Coronas-F/SPIRIT data will be available soon at the Web sites of the project participants.

\section{Acknowledgements}

We are grateful to Acad. I.I. Sobelman and Prof. V.D. Kuznetsov, scientific leaders of the CORONAS-F project, for the interest and support of this study; and to the Space Information Technology Center (IZMIRAN) for the assistance in the telemetry data supply. This work is supported by the RFBR (grants 02-02-17272 and 03-02-16591), the Federal Ministry of Science and Education (grants NSh 477.2003.2 \& 40.022.1.1.1104), and the program No. 18 of the OPhN RAS. 